\title{
https://helda.helsinki.fi
}

\section{Optimization of Tc-99m-sestamibi/l-123 subtraction SPECT/CT protocol for parathyroid scintigraphy}

\author{
Tunninen, V. \\ SPRINGER-VERLAG SINGAPORE PTE LTD \\ 2018
}

Tunninen , V , Kauppinen , T \& Eskola , H 2018 , Optimization of Tc-99m-sestamibi/l-123 subtraction SPECT/CT protocol for parathyroid scintigraphy . in H Eskola , O Väisänen , J Viik \& J Hyttinen (eds) , EMBEC \& NBC 2017 . IFMBE Proceedings , vol. 65, SPRINGER-VERLAG SINGAPORE PTE LTD , pp. 847-851, Joint Conference of the European Medical and Biological Engineering Conference (EMBEC) / Nordic-Baltic Conference on Biomedical Engineering and Medical Physics (NBC) , Tampere , Finland , 01/06/2017 . https://doi.org/10.1007/978-981-10-5122-7_212

http://hdl.handle.net/10138/327119

https://doi.org/10.1007/978-981-10-5122-7_212

acceptedVersion

Downloaded from Helda, University of Helsinki institutional repository.

This is an electronic reprint of the original article.

This reprint may differ from the original in pagination and typographic detail.

Please cite the original version. 


\title{
Optimization of ${ }^{99 \mathrm{~m}} \mathrm{Tc}$-sestamibi $/{ }^{123}$ I subtraction SPECT/CT protocol for parathyroid scintigraphy
}

\author{
V. Tunninen ${ }^{1}$, T. Kauppinen ${ }^{2}$ and H. Eskola ${ }^{3,4}$ \\ ${ }^{1}$ Satakunta Central Hospital/Department of Nuclear Medicine, Pori, Finland \\ ${ }^{2}$ Helsinki University Hospital/HUS Medical Imaging Center, Helsinki, Finland \\ ${ }^{3}$ Faculty of Biomedical Sciences and Engineering, Tampere University of Technology, Tampere, Finland \\ ${ }^{4}$ Deparment of Radiology, Tampere University Hospital, Tampere, Finland
}

\begin{abstract}
The purpose of this study was to optimize effective, but technically challenging ${ }^{99 \mathrm{~m}} \mathrm{Tc}$-sestamibi $/{ }^{123} \mathrm{I}$ subtraction SPECT/CT protocol for parathyroid scintigraphy. An anthropomorphic parathyroid phantom was set up using a small sphere, a thyroid phantom and a thorax phantom with clinical range of activities of ${ }^{123} \mathrm{I}$ and ${ }^{99 \mathrm{~m}}$ Tc. SPECT/CT acquisitions were performed using three collimators (Low Energy High Resolution (LEHR), Low Energy Ultra High Resolution (LEUHR) and Medium Energy Low Penetration (MELP)) and two energy window settings. Images were reconstructed with a combination of four different numbers of iterations and with or without scatter correction. Images were subjected to visual and quantitative evaluation. The effect of collimator, energy window selection and reconstruction parameters had a significant effect on visual appearance and adenoma contrast in parathyroid ${ }^{99} \mathrm{~m}$ Tc-sestamibi/ ${ }^{123}$ I subtraction SPECT/CT. Symmetrical energy windows and ultra-high resolution collimator yielded best results with some improvement with scatter correction.
\end{abstract}

Keywords - parathyroid, SPECT/CT, phantom, optimization, dual-isotope.

\section{INTRODUCTION}

The diagnosis of hyperparathyroidism is based on laboratory findings, with ionized calcium and parathormone levels being elevated. Patient should be referred for parathyroid scintigraphy only when above-mentioned diagnostic criteria are met. Parathyroid scintigraphy is thus not a diagnostic study, but a localization one. Localization of hyperfunctioning parathyroid glands before surgery is highly recommended to define surgical strategy.

The only tracer available for parathyroid scintigraphy, ${ }^{99 \mathrm{~m}} \mathrm{Tc}$-sestamibi is not parathyroid-specific but taken up also by adjacent thyroid tissue. This problem can be solved by using either a single isotope (dual-phase) or a dual-isotope method.

The dual-phase method assumes that thyroid and parathyroid tissue have different washout kinetics for ${ }^{99 \mathrm{~m}} \mathrm{Tc}$ sestamibi. By acquiring images immediately after the injection and 2-3 hours later, the focally increasing uptake will reveal hyperfunctioning parathyroid gland. In the dual- isotope method, ${ }^{99 \mathrm{~m}} \mathrm{Tc}$-sestamibi is used together with ${ }^{123} \mathrm{I}$, which is taken up by the thyroid gland only. Subtracting the ${ }^{123}$ I image from the ${ }^{99 \mathrm{~m}} \mathrm{Tc}$-sestamibi image leaves only enlarged parathyroid glands in the subtraction image. Imaging findings are based on a visual appearance of subtraction image, confirmed with anatomical reference in the CT image.

The advantages of dual-isotope method with ${ }^{99 \mathrm{~m}} \mathrm{Tc}$ sestamibi and ${ }^{123}$ I for parathyroid scintigraphy have been acknowledged in several studies and are recommended in the European Association of Nuclear Medicine (EANM) guideline for parathyroid scintigraphy [1]. This method has been applied in Finland, as all nuclear medicine departments performing parathyroid scintigraphy are now using dual-isotope method with ${ }^{99 \mathrm{~m}} \mathrm{Tc}$-sestamibi and ${ }^{123} \mathrm{I}$ as their first method of choice in parathyroid scintigraphy [2].

Both planar and tomographic acquisition techniques are used clinically. Planar pinhole acquisition has its advantages due to superior spatial resolution, but modern SPECT/CT imaging offers three-dimensional images together with exact anatomical information. These techniques are often combined to increase sensitivity and specificity [3-4]. However, acquisition of an additional image requires the change of the collimator and increases the total length of the study. Thus, it would be attempting to simplify imaging protocol but this has to be done without decreasing image quality. It should be noted that a large, active parathyroid adenoma is clearly visible regardless of the imaging protocol used. The current clinical problem are patients with secondary hyperparathyroidism with relatively inactive hyperplastic glands, and patients with multiglandular disease, where patient has more than one enlarged parathyroid glands [5].

${ }^{99 \mathrm{~m}} \mathrm{Tc}$-sestamibi $/{ }^{123}$ I subtraction SPECT/CT is technically challenging. Close proximity of the photon energies $(140 \mathrm{keV}$ and $158 \mathrm{keV}$ ) with limited energy resolution of a gamma camera detector leads to cross-contamination of isotopes. Asymmetric energy window for ${ }^{123} \mathrm{I}$ is generally used to reduce ${ }^{99 \mathrm{~m}} \mathrm{Tc}$ contamination in ${ }^{123} \mathrm{I}$ energy window, [6-7]. The septal penetration of high-energy photons of ${ }^{123} \mathrm{I}$ is also a potential cause of artefacts in subtraction images. The selection of collimator (due to different septal thick- 
ness, resolution and sensitivity) has thus a marked effect on the final results [8]. Reconstruction parameters and the use of scatter correction will also have effect [9-10].

The purpose of this study was to optimize the parathyroid scintigraphy ${ }^{99 \mathrm{~m}} \mathrm{Tc}-$ sestamibi $/{ }^{123} \mathrm{I}$ subtraction SPECT/CT - protocol using an anthropomorphic phantom. The effect of collimator choice, energy window settings and the reconstruction parameters (number of iterations, scatter correction) were studied.

\section{Materials AND METhods}

For the purpose of this study, a phantom mimicking parathyroid patient with a small, low intensity adenoma was set up (Figure 1). A thyroid gland phantom (Radiology Support Devices) was filled with ${ }^{123} \mathrm{I}$ and ${ }^{99 \mathrm{~m}} \mathrm{Tc}$ (concentration of $500 \mathrm{kBq} / \mathrm{ml})$. A small sphere with inner diameter of 7.86mm (Micro Hollow Sphere, Data Spectrum) was filled with lower ${ }^{99 \mathrm{~m}} \mathrm{Tc}$-concentration as thyroid gland $(\sim 300 \mathrm{kBq} / \mathrm{ml})$ to mimic small parathyroid adenoma or hyperplastic gland $\left(0.25 \mathrm{ml}\right.$ with $\sim 125 \mathrm{kBq}$ of $\left.{ }^{99 \mathrm{~m}} \mathrm{Tc}\right)$ and attached behind the thyroid gland. The thyroid and parathyroids were inserted into the thorax phantom (Radiology Support Devices), which was then filled with $350 \mathrm{MBq}$ of ${ }^{99 \mathrm{~m}} \mathrm{Tc}$. Activities were measured using a VIK-202 ionization chamber (Veenstra) used in clinical work and subjected to strict daily quality control measures.

A series of SPECT/CT scans were performed with three sets of collimators: Low Energy High Resolution (LEHR), Low Energy Ultra High Resolution (LEUHR) and Medium Energy Low Penetration (MELP) and with two energy window settings, clinical and test windows (Table I).

SPECT was acquired using normal clinical acquisition protocol (a noncircular orbit, $180^{\circ}$ detector configuration, $128 \times 128$ matrix; $4.8 \mathrm{~mm}$ pixel size; step-and-shoot, 48 views for each detector, $33 \mathrm{~s}$ /projection).

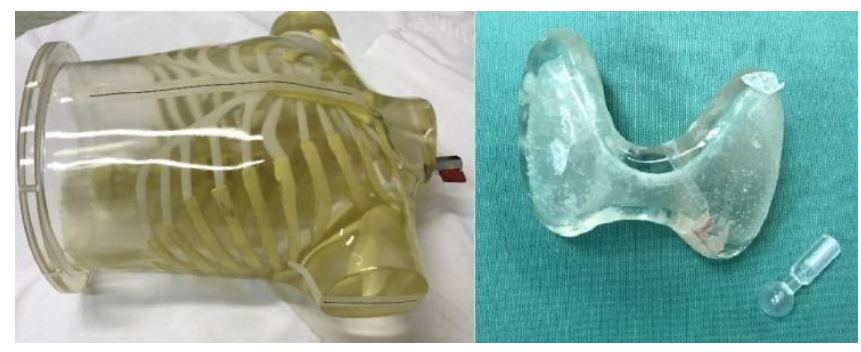

Figure 1. Thorax phantom, the thyroid gland and spherical phantom mimicking parathyroid adenoma. Images are not in scale.
Table 1 Energy window settings

\begin{tabular}{|c|c|c|c|c|}
\hline $\begin{array}{c}\text { Energy } \\
\text { Window } \\
\text { Setting }\end{array}$ & $\begin{array}{l}\text { Energy } \\
\text { Window }\end{array}$ & $\begin{array}{c}\text { Photopeak } \\
\text { at }\end{array}$ & $\begin{array}{c}\text { Window } \\
\text { Width } \\
(\%)\end{array}$ & $\begin{array}{c}\text { Window } \\
\text { Limits } \\
(\mathrm{keV})\end{array}$ \\
\hline \multirow{4}{*}{ Clinical } & Scatter & - & $15 \%$ & $112-133$ \\
\hline & ${ }^{99 \mathrm{~m}} \mathrm{Tc}$ & $140 \mathrm{keV}$ & $10 \%$ & $133-147$ \\
\hline & Scatter & - & $7 \%$ & $147-156$ \\
\hline & ${ }^{123} \mathrm{I}$ & $167 \mathrm{keV}$ & $10 \%$ & $159-175$ \\
\hline \multirow{4}{*}{ Test } & Scatter & - & $15 \%$ & $112-133$ \\
\hline & ${ }^{99 \mathrm{~m}} \mathrm{Tc}$ & $140 \mathrm{keV}$ & $10 \%$ & $133-147$ \\
\hline & Scatter & - & $2 \%$ & $147-149$ \\
\hline & ${ }^{123} I$ & $158 \mathrm{keV}$ & $10 \%$ & $150-165$ \\
\hline
\end{tabular}

The CT acquisition for attenuation correction and image fusion was acquired after the SPECT acquisition without moving the phantom and using the same scan area as for SPECT $(130 \mathrm{kVp}, 2 \times 2.5 \mathrm{~mm}$ collimation, $0.8 \mathrm{~s}$ rotation time, 1.5 pitch, CARE Dose AEC+DOM, with the ref. exposure $80 \mathrm{mAs})$. All acquisitions were performed with Siemens Symbia Intevo T2 SPECT/CT ((Siemens, Erlangen, Germany) in the Department of Nuclear Medicine of Satakunta Central Hospital.

${ }^{99 \mathrm{~m}}$ Tc-sestamibi and ${ }^{123}$ I SPECT images (with attenuation correction) were reconstructed on the Siemens Syngo workstation using the FLASH 3D-algorithm with eight parameter settings $(6,8,16$ or 32 iterations, 8 subsets, Gaussian 9.00 filter, with and without TEW scatter correction).

${ }^{123}$ I SPECT images were multiplied by a normalization factor (as the ratio of the thyroid maximum voxel counts in ${ }^{99 \mathrm{~m}} \mathrm{Tc}$ and ${ }^{123} \mathrm{I}$ SPECT images to create normalized ${ }^{123} \mathrm{I}$ SPECT images, which were then subtracted from ${ }^{99 \mathrm{~m}} \mathrm{Tc}$ SPECT images. All images were subjected to visual and quantitative evaluation. Image processing and evaluation was performed by an experienced medical physicist. All images were anonymized (no knowledge of collimator or acquisition or processing parameters) and scored from 0 to 5. Scoring was based on clinical appearance (the intensity of adenoma, the presence of artefacts).

For quantitative analysis, a VOI was drawn around the sphere using the fused CT image as a reference (with corresponding background VOI). An image contrast $\mathrm{C}$ for sphere was calculated using the formula (1):

$$
C=\frac{C_{\text {adenoma }}-C_{\text {background }}}{C_{\text {background }}} \times 100 \%
$$

where:

$\mathrm{C}_{\text {adenoma }}$ is the number of counts in the sphere,

$\mathrm{C}_{\text {background }}$ is the number of counts in the background area 
The mean values for contrast and visual scores were calculated for each collimator (all energy windows and processing parameters), for each energy window setting (all collimators and processing parameters) and for scatter correction (all collimators, energy windows and processing parameters).

\section{RESULTS}

The calculated contrast for adenoma was between $0,4 \%$ $80,9 \%$. The visual scores were between $0-5$. The contrast and visual scores were in correlation (Figure 2). The mean values for contrast and visual scores were highest for LEUHR collimator and for test energy windows settings. Contrast values were higher with scatter correction, but visual scores were lower (Table 2).

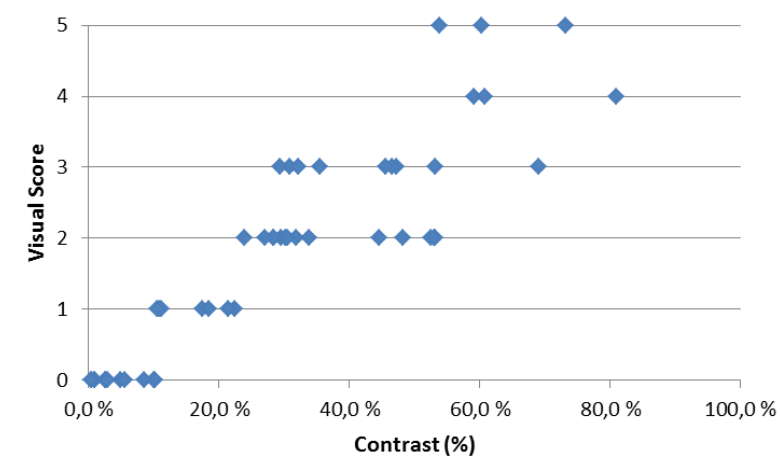

Figure 2. Visual score as a function of contrast.

Table 2 Mean contrast values and visual scores calculated for collimators, energy window settings and with or without scatter correction

\begin{tabular}{lcc}
\hline & Contrast $_{\text {mean }}$ & Visual $_{\text {mean }}$ \\
\hline MELP & $18 \%$ & 0,9 \\
LEHR & $26 \%$ & 1,8 \\
LEUHR & $47 \%$ & 2,9 \\
Clinical & $18 \%$ & 0,8 \\
Test & $43 \%$ & 2,9 \\
SC- & $28 \%$ & 1,9 \\
SC + & $32 \%$ & 1,8 \\
\hline
\end{tabular}

The mean values for contrast for reconstruction parameters (for all collimators and energy window settings) were higher with an increasing number of iterations. However, visual scores were highest with 16 iterations, increasing iterations to 32 decreased the visual appearance of the images (Table 3 ).

Table 3 Mean contrast values and visual scores calculated for eight reconstruction parameter settings for all collimators

\begin{tabular}{ccc}
\hline Iteration, SC-/SC+ & Contrast $_{\text {mean }}$ & Visual $_{\text {mean }}$ \\
\hline 6, SC- & $24 \%$ & 1,7 \\
$8, \mathrm{SC}-$ & $26 \%$ & 1,7 \\
16, SC- & $30 \%$ & 2,2 \\
$32, \mathrm{SC}-$ & $33 \%$ & 2,0 \\
6, SC+ & $22 \%$ & 1,5 \\
8, SC+ & $27 \%$ & 1,7 \\
$16, \mathrm{SC}+$ & $36 \%$ & 2,2 \\
$32, \mathrm{SC}+$ & $47 \%$ & 2,0 \\
\hline
\end{tabular}

The mean values for contrast for each combination of collimator, energy window and scatter correction are presented in Table 4. The highest mean values for contrast and visual scores were with LEUHR collimator with notcorrected test energy window. Phantom images with highest visual scores and with current clinical protocol are presented in Figure 3.

Table 4 Mean contrast values and visual scores calculated for collimators with two energy window settings and with or without scatter correction

\begin{tabular}{lcc}
\hline $\begin{array}{l}\text { Collimator, Energy Window, } \\
\text { Correction }\end{array}$ & Contrast $_{\text {mean }}$ & Visual $_{\text {mean }}$ \\
\hline MELP, Clinical, SC+ & $2 \%$ & 0,0 \\
MELP, Clinical, SC- & $9 \%$ & 0,0 \\
MELP, Test, SC- & $25 \%$ & 1,5 \\
MELP, Test, SC+ & $37 \%$ & 2,3 \\
& & \\
LEHR, Clinical, SC- & $9 \%$ & 0,5 \\
LEHR, Clinical, SC+ & $9 \%$ & 0,5 \\
LEHR, Test, SC- & $30 \%$ & 2,5 \\
LEHR, Test, SC+ & $55 \%$ & 3,5 \\
& & 2,5 \\
LEUHR, Clinical, SC- & $30 \%$ & 2,0 \\
LEUHR, Clinical, SC+ & $50 \%$ & 4,8 \\
LEUHR, Test, SC- & $67 \%$ & 2,8 \\
LEUHR, Test, SC+ & $4 \%$ & \\
\hline
\end{tabular}




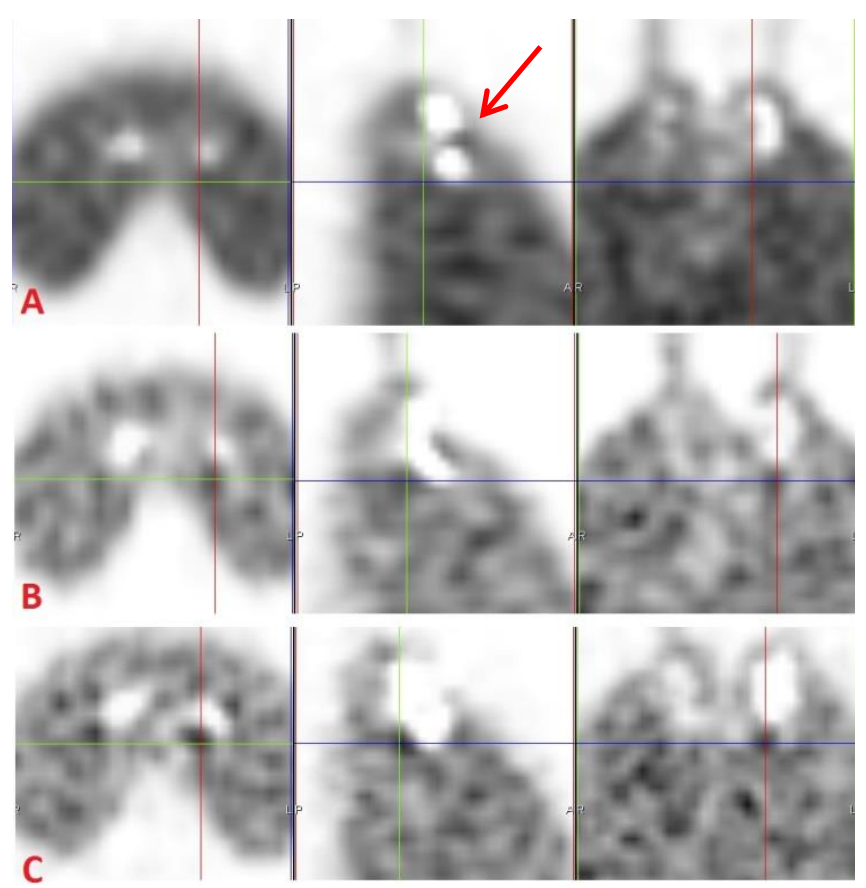

Figure 3. From left to right, transverse, sagittal, and coronal images of the phantom (an adenoma is located at the crosshair). A) LEHR Clinical, 8 iterations, no scatter correction (current clinical protocol), B) LEHR Test,

16 iterations, scatter correction, and C) LEUHR Test, 16 iterations, no scatter correction. The red arrow is showing "the edge artefact".

\section{DisCUSSION}

The findings of this study confirmed that the selection of collimator, energy window positioning and reconstruction parameters have a significant effect on the visual appearance of images. Collimators with higher resolution yielded better results, as did increasing iterations in reconstruction. The energy window setting had a significant effect on the appearance of subtraction images, as symmetric window for ${ }^{123} \mathrm{I}$ was always better than asymmetric window. Scatter correction increased calculated contrast, but at the same time, the visual appearance was decreased due to increased noise.

Current clinical protocol for parathyroid scintigraphy with LEHR collimator, asymmetric energy window without scatter correction was able to visualize adenoma only faintly. Switching to symmetric energy window for ${ }^{123} \mathrm{I}$ and applying scatter correction with more iterations was able to increase visual score from 0,5 to 3,5. MELP collimator would be attempting choice for imaging with ${ }^{123} \mathrm{I}$ due to thicker septa, but low spatial resolution is not adequate when searching for small, relatively inactive parathyroid gland. LEUHR collimator with symmetric energy windows had best contrast and visual scores. The scatter correction in fact decreased these results, probably due to statistics as LEUHR collimator has sensitivity of approximately $50 \%$ compared to LEHR collimator and subtraction-based scatter correction reduces counts even further. The visual effect of increasing noise is visible in the Figure 3, where the artifactual "blobs" have a similar appearance to parathyroid adenoma. However, this misinterpretation can be avoided with the use of CT, and the search of the anatomical reference.

The so-called edge artefact [11] is currently the main cause of false positive findings in ${ }^{99 \mathrm{~m}} \mathrm{Tc}$-sestamibi $/{ }^{123} \mathrm{I}$ subtraction SPECT/CT (Figure 3). According to the results of this study, the edge-artefact is more present with lower resolution image and with asymmetric ${ }^{123} \mathrm{I}$ window. LEUHR symmetric window images did not include any marked artefact.

According to the results of this study, increasing the number of iterations to more than 16 decreased the visual scores due to increased noise. However, it should be noted that this is highly dependent on camera and collimator specifications, acquisition parameters and activity of ${ }^{99 \mathrm{~m}} \mathrm{Tc}-$ sestamibi and ${ }^{123}$ I used.

Due to large variation of adenoma contrast and visual scores in this study, we find that the protocols used in clinical practice should be thoroughly tested using phantoms. Although clinical situation is different when comparing to phantom studies (patient movement, breathing, extra thyroid ${ }^{99 m}$ Tc-sestamibi activity), the ultimate detection limit should be resolved.

\section{v. Conclusions}

The effect of collimator, energy window selection and reconstruction parameters have a significant effect on visual appearance and adenoma contrast in parathyroid ${ }^{99 \mathrm{~m}} \mathrm{Tc}$ sestamibi $/{ }^{123}$ I subtraction SPECT/CT. Symmetrical energy windows and high-resolution collimator yielded best results with some improvement with scatter correction.

\section{CONFLICT OF INTEREST}

The authors declare that they have no conflict of interest.

\section{REFERENCES}

1. Hindie E, Ugur O, Fuster D, et al (2009). 2009 EANM parathyroid guidelines. Eur J Nucl Med Mol Imaging; 36:1201-1216.

2. Tunninen V, Varjo P, Kauppinen T. Towards harmonization in parathyroid scintigraphy in Finland - A seven year follow-up study (2016). EJNMMI 43; Suppl 2. 
3. Bahador FM, Latifi HR, Grossman SJ, et al (2015). Optimal interpretative strategy for preoperative parathyroid scintigraphy. Clin Nucl Med;40:116-122

4. Hassler S, Ben-Sellem D, Hubele F, et al. (2013). Dual-Isotope ${ }^{99 \mathrm{~m}} \mathrm{Tc}-\mathrm{MIBI} /{ }^{123} \mathrm{I}$ Parathyroid Scintigraphy in Primary Hyperparathyroidism: Comparison of Subtraction SPECT/CT and Pinhole Planar Scan. Clin Nucl Med; 39(1):32-6

5. Hindie E, Zanotti-Fregonara P, Tabarin A, et al (2015). The Role of Radionuclide Imaging in the Surgical Management of Primary Hyperparathyroidism. J Nucl Med;56:737-744

6. Neumann DR, Obuchowski NA, Difilippo FP (2008). Preoperative ${ }^{123} \mathrm{I} /{ }^{99 \mathrm{~m}} \mathrm{Tc}-\mathrm{Sestamibi}$ Subtraction SPECT and SPECT/CT in Primary Hyperparathyroidism. J Nucl Med;49:2012-2017.

7. Dontu VS, Kettle AG, O'Doherty MJ, et al. (2004). Optimization of parathyroid imaging by simultaneous dual energy planar and single photon emission tomography. Nucl Med Commun ;25:1089-1093

8. Inoue Y, Shirouzu I, Machida T, et al. (2003). Physical characteristics of low and medium energy collimators for ${ }^{123} \mathrm{I}$ imaging and simultaneous dual-isotope imaging. Nucl Med Comm (24); 1195-1202.
9. Nichols KJ, Tronco GG, Palestro CJ (2015). Effect of reconstruction algorithms on the accuracy of $\left({ }^{99 m}\right) \mathrm{Tc}$ sestamibi SPECT/CT parathyroid imaging. Am J Nucl Med Mol Imaging. 5:195-203

10. van Hoorn RA, Vriens D, Postema JW, et al. (2013). The influence of SPECT reconstruction algorithms on image quality and diagnostic accuracy in phantom measurements and ${ }^{99 \mathrm{~m}} \mathrm{Tc}-$ sestamibi parathyroid scintigraphy. Nucl Med Commun; 35; 6472.

11. Tunninen V, Varjo P, Schildt J, et al (2013). Comparison of Five Parathyroid Scintigraphic Protocols. Int J Mol Imaging, Article ID 921260, 12 pages, 2013. doi:10.1155/2013/921260

Author: Virpi Tunninen, Chief Physicist Institute: Satakunta Central Hospital,

Department of Nuclear Medicine

Street: Sairaalantie 3

City: 28500 Pori

Country: Finland

Email: virpi.tunninen@satshp.fi 